\title{
LE RÉENCHANTEMENT DU RÉCIT RADIOPHONIQUE COMME RÉENCHANTEMENT DU MONDE
}

\begin{abstract}
Sébastien Poulain ${ }^{1}$
Cet article consiste principalement à décrire et analyser la place et la contribution du récit de la radio New Age Ici et Maintenant au sein des phénomènes d'enchantement, de désenchantement et de réenchantement du monde.

Il s'agira de se demander comment l'enchantement ou le réenchantement du récit radiophonique peut conduire à une forme d'enchantement ou de réenchantement du monde. L'hypothèse de départ est que ce qui a été qualifié de fin progressive des " grands récits » est consubstantielle à une forme de « désenchantement du monde » qui trouve des formes de compensation par l'apparition de « petits récits » réenchanteurs comme celui diffusé par la radio associative parisienne Ici et Maintenant (RIM). En effet, cette radio tente de réenchanter ce récit par la propagation de ses idées New Age tant du point de vue de sa forme discursive que de son contenu politico-religieux.
\end{abstract}

Le réenchantement du politique consiste principalement à présenter autrement l'actualité politique. Il y a réenchantement dans la manière de parler de politique et dans l'interprétation qui en est faite.

1 Sébastien Poulain est doctorant au laboratoire MICA, Université de Bordeaux 3.

Recherches en communication, $\mathrm{n}^{\circ} 37$ (2012). 
RIM a une manière d'aborder l'actualité spécifique à certaines « radios libres » (Poulain, c) et associatives qui voulaient ou veulent redonner la parole aux citoyens dans la lignée de l'utopie communicationnelle deleuzoguattarienne. Elle donne depuis 30 ans de nombreuses heures d'antenne à ses auditeurs dans des émissions intitulées « Antenne libre » (la nuit) ou « Revue de presse interactive » (en début d'aprèsmidi) (Poulain, 2008). Les auditeurs peuvent y intervenir (jusqu'à trois simultanément) sans aucune sélection préalable par un standard en donnant simplement leur prénom. Le principe consiste à laisser une liberté absolue aux appelants (sauf exceptions légales 1 ). Il s'agit de laisser cours au pluralisme et à l'inattendu. Les auditeurs sont là pour donner simplement leur avis sincère qui pourra d'ailleurs évoluer au fur et à mesure de la discussion socratique avec les autres auditeurs, les animateurs et les invités. Pour cela, ils peuvent rester jusqu'à 20 minutes (surtaxées) pour décortiquer, interpréter et réinterpréter l'actualité et l'histoire politique, économique, écologique... Chacun peut ajouter sa petite pierre narratologique à l'édifice discursif radiophonique.

Pour les promoteurs de RIM, ce type de dispositif (que l'on retrouve sur d'autres antennes avec intervention d'auditeurs) est la seule façon d'atteindre la sincérité, l'objectivité, la neutralité, donc la vérité, car les auditeurs qui passent à l'antenne ne sont pas censés appartenir à une collectivité, un groupe, une institution, donc défendre une entreprise, un parti politique ou un lobby. Il n'y a pas d'enjeux économiques et politiques sous-jacents ni de conflit d'intérêt possible car les auditeurs ne semblent représenter qu'eux-mêmes du fait de leur anonymat et de leur invisibilité. Chaque auditeur appelant est considéré comme un témoin de la réalité avec une légitimité a priori égale à celles des autres auditeurs mais aussi des invités et animateurs (même si les inégalités en termes de capitaux symboliques et culturels font la différence). Et cet effet est accentué par le statut non commercial et non-public de la radio que les auditeurs saluent souvent.

$\mathrm{Si}$, dans la réalité quotidienne, certains auditeurs se sentent impuissants en raison de leurs problèmes relationnels, de solitude, de deuil, de santé et de travail, et s'ils estiment la réalité trop éloignée de leurs idéaux, ils peuvent utiliser la magie du verbe, décuplée par le média, pour amplifier en quelque sorte leur «puissance » d'action. " Au commencement était le Verbe » (Prologue de l'évangile selon Jean,

1 RIM a perdu sa fréquence entre 1996 et 2001 suite à des propos racistes et antisémites d'un auditeur. 
verset 1), donc les auditeurs peuvent profiter de leur passage à l'antenne pour retourner aux origines du monde et le recommencer. Chacun peut modeler et remodeler, déconstruire et reconstruire à volonté la réalité. Nuit et jour, ils défont et refont le monde comme les dieux de l'Olympe. La réalité étant souvent jugée décevante, insuffisante, sèche, triste, froide, désenchantée, il s'agit donc d'inventer d'autres manières de la raconter pour l'améliorer. Si la réalité ne peut pas changer immédiatement, il est possible de changer la manière de la penser et de la raconter. Les animateurs, invités et auditeurs de RIM peuvent faire une exégèse infinie de la réalité pour la revivifier et la réenchanter en s'appuyant sur l'effet « cathartique » et « performatif » (Derville, 1995) du récit.

A côté de déclarations de soutien et de solidarité à l'antenne qui font de la radio un lieu de socialisation où il est possible d'aborder des sujets « tabous » dans ses cercles familiaux, amicaux ou professionnels à cause de la peur de la moquerie, du conflit ou de l'indifférence, on peut trouver beaucoup de témoignages, d'interrogations, de réflexions, de conseils ${ }^{1}$ et d'idées pour élaborer des politiques (économiques, sociales, fiscales, environnementales) alternatives à celles mises en place par les gouvernements successifs. C'est souvent aussi un déferlement de procureurs contre-experts qui semblent régler leurs comptes avec la société (leur passé, leur famille, les politiques, les employeurs...). À chaque moment, avec chaque nouvelle personne qui passe à l'antenne, tout peut être remis en question. Personne ne peut avoir le dernier mot, le monopole du récit et de la légitimité discursive. Tout peut être débattu, réexpliqué, retraduit, recodifié par cette communauté d'interprétation plurielle. La seule vérité est « ici et maintenant» dans le discours émis à l'antenne qui devient en quelque sorte la seule réalité au moment où il est prononcé.

Mais en quoi il y a-t-il réenchantement du politique à l'antenne de RIM ? Quelle est la réalité politique décrite?

RIM n'a jamais été liée à un parti politique. Les femmes et hommes politiques y interviennent rarement en dehors des élections présidentielles où les auditeurs ont pu interroger Governatori, DupontAignant, Marine et Jean-Marie Le Pen... Aucune association militante

1 L'émission "Sante et spiritualité » de fin d'après-midi (et certaines émissions nocturnes) où des nutritionnistes, voyants, astrologues... viennent donner des conseils alimentaires, thérapeutiques, psychologiques, spirituelles transforment l'antenne en radio du « divan » (Deleu, 2006). 
ne dispose d'un temps d'antenne comme cela peut être le cas dans d'autres radios associatives ${ }^{1}$. Des actions politiques (pétitions, manifestations...) découlent rarement directement des émissions. Les animateurs ne se considèrent pas comme des militants politiques mais comme des animateurs-journalistes neutres.

Néanmoins, RIM invite quotidiennement des représentants d'associations militantes écologistes, anticonsuméristes, anticapitalistes, qui peuvent eux-mêmes inviter les auditeurs à rejoindre leurs combats, leurs manifestations, à signer des pétitions, à les soutenir économiquement pour combattre les inégalités, les injustices, les illégalités, les discriminations, les dangers environnementaux et sanitaires... Et ces représentants ont bien été choisis en fonction de l'idéologie écolo-biospirituelle que porte la radio.

Mais RIM fait aussi de la politique par le simple fait de «montrer » le pouvoir - qu'il soit politique, militaire, industriel, financier... - en partant du postulat que d'autres médias n'en ont pas la possibilité du fait de leur dépendance vis-à-vis des subventions publiques ou de la publicité. Cette radio souhaite montrer comment s'exercent les rapports de domination et comment le pouvoir et ses élites essayent de cacher leur façon de se différencier, de s'entraider, de se reproduire. Il n'est donc pas étonnant qu'elle ait pu inviter Monique Pinçon-Charlot et Michel Pinçon à l'occasion de la parution du Président des riches (2010) dont la sociologie bourdieusienne ne pouvait qu'intéresser les animateurs et auditeurs.

Face à l'usage tous azimuts des stratégies de communication et du storytelling des communicants, des publicitaires, des journalistes et des politiques, les animateurs, invités et auditeurs de RIM souhaitent proposer des " ressources cognitives » (conatives, symboliques, psychologiques) alternatives pour essayer de résister. Il s'agit de donner la possibilité d'interpréter et réinterpréter, déconstruire et reconstruire les discours dominants des représentants politiques, institutionnels, économiques, à l'image du travail de lecture critique de la constitution européenne par Étienne Chouard (plusieurs fois invité à l'antenne) en 2005. À RIM, la réalité, telle qu'elle est racontée dans la société et la plupart des médias, est remplie de mensonges et manipulations. La

1 Les animateurs sont souvent recrutés parmi les auditeurs car l'adhésion à une grande partie de l'idéologie, de la culture et de l'organisation de la radio est un préalable (y compris, mais peut-être dans une moindre mesure, pour les animateurs invités au micro grâce à leur expertise). 
liberté laissée à l'antenne est donc l'occasion de le dire et le démontrer afin de rétablir la vérité, dénoncer la communication requalifiée en propagande étatique et capitaliste, lancer ou relayer diverses rumeurs (l'absence de sortie lunaire en 1969, l'absence de crash d'avion sur le Pentagone en $2001^{1} \ldots$...).

Avec son regard critique, RIM essaye de montrer ce que le pouvoir dit et fait, autant que ce qu'il ne dit pas et ne fait pas alors qu'il le devrait. Elle compare les objectifs, les programmes et leurs résultats. C'est ainsi qu'André-Jacques Holbecq (ancien pilote du Concorde qui s'est vanté par ailleurs de recevoir des lettres des extraterrestres de la planète Ummo et a créé le site ummo-sciences.org sur ce sujet) avait animé la « Revue de presse interactive » en décryptant les programmes économiques des candidats aux présidentielles de 2007 à la lumière de l'écosociétalisme qu'il promeut.

Mais RIM va au-delà encore dans sa manière de montrer le pouvoir. Elle approfondit toujours plus sa critique en montrant ce qui se fait sans se dire et ce qui se dit sans se faire pour dénoncer les disjonctions entre le dire et le faire. RIM se différencie des médias traditionnels dans sa manière de systématiser le doute sur les activités politiques, militaires et économiques des institutions publiques. La parole politique serait mensongère, manipulatrice, malfaisante par nature et non par occasion. Ainsi, la grille d'interprétation de la politique adoptée est souvent complotiste : la totalité de la réalité passe de l'officiel à l'officieux et de l'exotérisme à l'ésotérisme². Le monde serait gouverné par

1 Antoine Marette (France Culture) était venu interviewer Meyssan à propos du « pentagate» le mardi 17 septembre 2002.

2 A RIM, ce sont souvent les mêmes animateurs, invités ou auditeurs qui traitent avec le même raisonnement ésotériste un sujet politique la journée et un sujet spirituel la nuit. Ainsi, l'animateur Laurent Fendt, issu des Beaux arts de Versailles, anime «Antenne libre » de nuit (le dimanche à partir de 23h) traitant surtout de questions philosophiques et spirituelles. Mais il a pu y inviter Pierre Hillard (docteur en science politique et professeur de relations internationales à l'ESCE) venu pour traiter et critiquer les processus technocratiques de « décomposition » des nations et d'unification du monde dirigés par des « blocs continentaux unifiés » à l'instar du bloc « euro-atlantique ». Laurent Fendt anime aussi l'émission "Santé et spiritualité » traitant de spiritualité, de médecines alternatives, d'ésotérisme ou de voyance. Il co-anime encore l'émission « La Revue de Presse Internationale » (un mercredi sur deux à 14 h) traitant de la crise économique et financière avec Pierre Jovanovic. Ce dernier est un ancien journaliste du Matin de Paris puis du Quotidien de Paris. Il est aussi l'auteur d'Enquête sur l'existence des anges gardiens et de 777, la chute du Vatican et de Wall Street selon Saint Jean où il développe la thèse d'une banqueroute 
une minorité de comploteurs, c'est-à-dire des personnes qui prennent et conservent le pouvoir (et l'argent) de façon illégale et immorale dans leur intérêt et non celui des personnes qu'ils gouvernent. Il s'agit alors, pour la communauté RIM, de relocaliser les lieux où est « réellement » détenu le pouvoir. Si officiellement, ces lieux se trouvent au sein des institutions exécutives, législatives et judiciaires, le pouvoir serait officieusement ailleurs, dans des sociétés et organisations secrètes non démocratiques et transnationales : le Groupe Bilderberg, la Commission Trilatérale, le Club de Rome... Pour justifier leurs critiques, ils peuvent facilement s'appuyer sur la complexité et l'absence de lisibilité du champ politico-économique contemporain. En effet, les phénomènes de mondialisation, européanisation et décentralisation ont provoqué la multiplication d'institutions régionales, nationales, internationales ou transnationales dites de " gouvernance " plus ou moins démocratiques et concurrencées par des entreprises internationales plus riches que les États, donc susceptibles de peser sur leurs décisions et législations grâce à leur travail de lobbying. RIM se propose de revisiter l'actualité et l'histoire politique pour en dévoiler les ressorts et les intérêts cachés. Elle « intentionne » ou plutôt " réintentionne » les hommes politiques. En effet, alors que ces derniers ont tendance à se présenter traditionnellement dans une posture altruiste de « don de soi » au nom de l'intérêt général et du service public, RIM remet en doute ce discours et dénonce la privatisation de la chose publique et les mauvaises intentions cachées derrière les apparences.

La politique est d'une certaine façon réenchantée par RIM dans la mesure où les gouvernements et les institutions officiels seraient conduits par d'autres volontés qui les transcenderaient et les dépasseraient : celles de personnes cachées dans des lieux cachés et ayant des intérêts cachés. Cette vision donne à la réalité une dose de mystère qui peut faire réfléchir et donner envie d'effectuer des recherches sur cet ésotérisme politique. Le récit complotiste, que l'on peut qualifier de science politique profane anti-institutionnelle, est bien une forme de réenchantement du récit, mais c'est un réenchantement désenchanteur puisque la réalité politique est présentée comme sclérosée, fermée, factice, corrompue. L'enchantement se transforme donc rapidement en dégoût ou approfondit une aversion préexistante. Si le récit radiophonique sur la politique permet une grande liberté d'expression, il ne peut

universelle jumelée à la chute du Vatican en se fondant sur l'Apocalypse de Saint Jean. 
à lui seul redonner espoir pour changer une société qui semble si figée. Ce n'est pas ou plus sur la scène politique que se jouent les choses essentielles pour le bien de l'humanité. Le réenchantement radiophonique en matière de politique fourni par RIM ne suffit pas à la demande de réenchantement des animateurs, invités et auditeurs qui aspirent à d'autres formes de respiration et d'inspiration.

Mais le réenchantement du politique peut compter sur le réenchantement du religieux comme forme d'action politique par d'autres moyens (Dozon, 2008). Si le réel offre peu de possibilités de sortie, alors pourquoi ne pas rechercher des solutions dans l'« irréel ». Nous allons voir que RIM tente de déstabiliser voire déstructurer les frontières entre visible et invisible, normal et « paranormal », foi et raison, vrai et

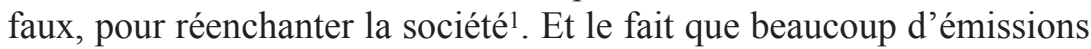
de RIM aient lieu la nuit entre « écoute en veilleuse » et « écoute éveilleuse » (Sorbets, 2003) peut aider. Selon un auditeur, l'écoute nocturne permet de « suspendre son incrédulité » et "surinterpréter une réalité possible », donc de s'ouvrir à d'autres formes de récit mélangeant science, fiction, politique et spiritualité à l'image du « merveilleux technologique » ufologique promu par la radio le mardi soir à $23 \mathrm{~h}^{2}$. Selon le même auditeur, le récit proposé est « à la fois hallucinant et hyper crédible » ce qui rejoint le concept d'Alain Gauthier (1996) de « temps symbolique » présenté comme une « force irrépressible qui transporte le sujet, le ravit de ses certitudes ou convictions, qui désespérément l'enchante ».

\section{Réenchantement du religieux}

1 Didier de Plaige, cofondateur et président de RIM, est l'auteur du roman cyberpunk Protocole oracle (Chamanéditionuméric, 2012) traitant des liens entre le monde politico-financier et la précognition obtenue grâce à l'utilisation d'une formule chimique classifiée. Les dirigeants de la " fédération » font discrètement procéder, avant chaque décision importante, à une séance de " voyages dans la conscience " financée grâce aux fonds secrets de la présidence. Les droits d'auteur de cet ouvrage serviront au projet Radio-Shipibo - parrainé par Jan Kounen - qui vise à doter les communautés shamaniques péruviennes Shipibo-Conibo - qui utilisent l'Ayahuasca (un breuvage à base de lianes aux effets purgatifs et hallucinogènes) pour la transe et la thérapie - de radios solidaires et contestataires.

2 Dans les années 2000, Plaige s'est beaucoup intéressé aux OVNI. Il produit et présente une émission sur le sujet : «La vague d'OVNIs » et a créé le blog ovnisusa.com sur son actualité quotidienne aux Etats-Unis et ailleurs. 
Face à une société contemporaine décrite en termes de « désenchantement du monde », RIM effectue un travail de réenchantement religieux à travers la promotion de ce qu'on peut appeler, pour simplifier, la « pensée New Age ».

Le concept de "réenchantement ", que l'on retrouve dans les philosophies sociales postmodernes ${ }^{1}$ de Michel Maffesoli et Bernard Stiegler, semble aller à l'encontre de ce que Max Weber puis Marcel Gauchet ont appelé le "désenchantement du monde ». Pour Weber, qui se place d'un point de vue sociologique, ce « désenchantement» correspond au déclin de la magie et des religions en tant que technique de salut et vision du monde qui se manifeste par le fait que les individus diminuent leurs pratiques, valeurs et croyances religieuses. Pour Gauchet, qui se situe d'un point de vue davantage philosophique et politique, ce « désenchantement » correspond au fait que la collectivité humaine n'est plus dirigée selon les prescriptions de la transcendance divine interprétées par les institutions religieuses ou politico-religieuses (l'exemple le plus manifeste étant la monarchie absolue de droit divin française) mais par des institutions laïques. Il y a "démocratisation » (Poulain, a) du " champ religieux », c'est-à-dire démonopolisation du pouvoir symbolique des institutions religieuses jusqu'à présent en charge de la gestion du réel et de "l'économie des biens du salut » (Bourdieu, 1971).

Avant les philosophes des Lumières qui ont porté une idéologie matérialiste, humaniste et progressiste visant à provoquer ce « désenchantement » par le développement de la science moderne (prosaïsme, scientisme, matérialisme, rationalisme) mais aussi des évolutions socioéconomiques et politico-juridiques, le fait de ne pas avoir de réponses à toutes nos questions métaphysiques sur le " pourquoi » des choses est relativement acceptable dans la mesure où le monde est « enchanté » par le divin. Une volonté divine monothéiste ou polythéiste dirige le monde sans partage ou presque. Les choses sont telles qu'elles sont parce qu'elles sont voulues ainsi par le divin qui imprègne tout ce qui existe. Les choses, animaux, événements et personnes extraordinaires (saints, miracles, monstres, prodiges...) sont interprétés comme des manières de communiquer, punir ou récompenser les humains. L'optimisme - ou

1 Sur les liens entre la postmodernité, la postradio et RIM : Poulain, b. 
fatalisme - leibnizien, si bien caricaturé par Voltaire dans Candide, rend tolérable le mal comme une étape eschatologique nécessaire vers le salut. Dans le débat aristotélicien sur ce qui relève de la contingence ou de la nécessité, c'est la nécessité divine qui a pris le dessus. Le rationalisme religieux (Thomas d'Aquin, Augustin...) domine le rationalisme scientifique politiquement autant que scientifiquement.

Mais l'idéologie des Lumières tend à rationaliser et matérialiser tout ce qui existe. Tout peut être mesuré, chiffré, calculé... Tout devient logique et scientifique. Il y a rationalisation et chosification du monde. Le marxisme, le positivisme et le structuralisme (voire la phénoménologie) servent de paradigmes pour déceler l'organisation du monde. Il s'agit de voir que les choses, les faits, la matière, les structures organisent le monde. La société est autant atomisée par les sciences humaines que par les sciences naturelles. Selon les sciences humaines, notamment chez Durkheim, 1'homme en tant qu'être social est travaillé par la puissante force de l'individualisme moderne qui semble créer une société remplie d'êtres libres et autonomes mais désocialisés et anomiques. Selon les sciences naturelles (biomédicales), l'homme en tant qu'être biologique est analysé comme un ensemble de gênes, atomes, organes dont la congruence réticulaire permet le fonctionnement.

Que la réalité soit atomisée par les scientifiques ou divinisée par les religieux, il s'agit toujours d'abolir le hasard en rationalisant le monde avec deux idéologies différentes mais en partant du postulat que les choses sont logiques, voire téléologiques. Toutefois la science essaye de découvrir la logique des choses sans être certaine de pouvoir atteindre ce qui est à l'origine de cette logique, alors que la religion se concentre sur ce qui est à l'origine de cette logique en accordant bien moins d'importance à la manière précise et concrète dont les choses se réalisent. La science accepte la possibilité de ne pas avoir les moyens de donner des réponses sur les causes premières tandis que la religion donne d'emblée des réponses à ces questions.

Dans leur manière de tenter d'abolir le hasard, les deux logiques sont confrontées à ce qu'on pourrait appeler la nature « diabolique » (du grec ancien diaballein, lancer à travers, calomnier, diffamer, diverger, séparer) de la nature. En effet, cette nature semble parvenir à fonctionner (vivre) tout en étant d'apparence chaotique et illogique donc incompréhensible et imprévisible. Dans la nature, les choses paraissent divisées, éparpillées, uniques, irrationnelles. Elles existent sans qu'on puisse y voir un sens évident et immédiat. Elles vivent autour de nous sans qu'on puisse communiquer avec elles ni les contrôler malgré un 
travail titanesque de domestication et de technicisation du monde. Le monde peut sembler gouverné par le hasard et l'absurde, que l'on se situe au niveau de l'infiniment grand ou de l'infiniment petit, voire à taille humaine : l'imprévisibilité de la météorologie, la diversité des paysages, des climats, de la faune et de la flore, les processus physicochimiques et géologiques (éruptions volcaniques, secousses sismiques, mouvements tectoniques, flux thermiques...).

La probabilité que la terre et l'homme puissent exister semblait donc très faible ${ }^{1}$. Et cette curieuse unicité de la place de l'homme dans l'univers, combinée à sa curiosité, l'enjoignent à une recherche ontologique infinie dans la mesure où il n'a pas a priori de réponses aux questions sur le sens de son existence et de l'univers. Face à la nature (c'est-à-dire la physique selon l'étymologie grecque physis), l'homme réalise un travail " méta-physique » et "symbolique » (du grec ancien symballein, rassembler, unir, joindre) à travers un discours, des idées, une pensée structurée (une idéologie) sur cette nature dont il fait partie. Il organise, ordonne, construit le sens des choses par le langage juridique, économique, physique, chimique, mathématique, religieux... Qu'elle soit religieuse ou scientifique, la symbolisation (le discours métaphysique) vise à remettre en ordre le " chaos » « diabolique » du monde. Grâce au langage, les hommes créent des liens entre des choses qui n'en n'ont pas forcément en apparence (astronomie) ou pas du tout (astrologie). Ces liens discursifs permettent de prendre les choses ensemble, c'est-à-dire de les com-prendre. C'est en cela que l'homme est un « enchanteur ». Il fait " chanter» (même origine étymologique qu' « incantation ») les choses en les harmonisant et les vitalisant par le sens qu'il leur donne. Le récit étant une forme d'enchantement du réel, le réenchantement du récit permet à l'homme de redonner vie à sa manière de raconter la vie. Si le « désenchantement du monde » sépare, éparpille, divise, " diabolise » les choses car le discours religieux traditionnel perd son emprise sur le réel sans que le discours scientifique parvienne à prendre le relai, le travail d'enchantement ou de réenchantement tente de les rassembler, unir, organiser grâce à l'injonction de nouvelles formes symboliques.

1 Les ufologues connaissent bien l'équation de l'astronome Frank Drake qui vise à calculer la possibilité qu'une civilisation semblable à celle des terriens existe : $\mathrm{N}=$ $R \times f_{p} \times n_{e} \times f_{1} \times f_{i} \times f_{c} \times L$. 
Pour réinjecter du symbolique donc réenchanter le monde, RIM promeut la « pensée New Age » en invitant de « nouveaux prophètes » ${ }^{1}$ - médiums, astrologues, guérisseurs, énergéticiens et autres thérapeutes - porteurs de cette idéologie censée « éveiller » les « consciences » de l'humanité.

Le New Age (Ferreux, 2000) est un courant de pensée qualifié de « nébuleuse-mystique-ésotérique » par Françoise Champion appartenant à ce qui a été appelé les « Nouveaux Mouvements Religieux » ou « Mouvements Religieux Contemporain » et qui prophétise l'avènement d'un Nouvel Age d'harmonie universelle, de paix, d'amour, de bonheur, de spiritualité appelé « ère du Verseau » ou " conspiration du Verseau ». Ce dernier doit remplacer 1' « ère du poisson », faite de guerres, violences, haines...) qui a député en 1413 et doit s'achever en 3573 selon l'anthroposophe Steiner. Ce courant de pensée est apparu au début des années 1960 en Californie. Il adapte des philosophies et religions orientales (notamment le bouddhisme tibétain ${ }^{2}$ ) et occidentales (notamment la tradition spiritualo-ésotérique ${ }^{3}$ ) aux mouvements de la contre-culture américaine ${ }^{4}$. C'est, selon Françoise Champion, un

1 Les nouveaux prophètes (Paris : Buchet-Chastel) est le titre de l'ouvrage, écrit par Plaige et Jean-Marie Leduc en 1978, qui recense et décrit les «mouvements religieux » qui ont émergé ou sont en train d'émerger dans les années 1960-1970.

2 Une partie importante des premiers animateurs de RIM, d'origine catholique ou juive, se sont convertis au bouddhisme. Plaige a été professeur de yoga en Ecosse et a participé financièrement à la fondation du Temple des mille Bouddhas en Bourgogne.

3 La majorité des animateurs se sont intéressés aux différentes traditions spirituelles et ésotériques. "Gurdjieff » est le nom d'une chanson écrite et interprétée par Guy Skornik (cofondateur de la radio) en hommage à une figure célèbre de l'ésotérisme : Georges Gurdjieff. Jean-Paul Bourre (animateur depuis 1982), qui a été rédacteur en chef de la revue d'ésotérisme L'Autre Monde, a écrit des ouvrages sur le satanisme aussi bien que sur la sorcellerie et le druidisme. Chaque jour, de $6 \mathrm{~h}$ à $7 \mathrm{~h}$ et en anglais, RIM diffuse une émission à partir des livres audio (comme The Power of Now) du penseur spirituel allemand Eckhart Tolle (une référence au mystique rhénan Maître Eckhart qui invitait à la déshabitation du corps pour s'unir à Dieu).

4 Plaige a traduit le best-seller New Age de Richard Alpert Be Here Now publié en 1971 aux Etats-Unis. Richard Alpert, dit Ram Dass, avait été professeur de psychologie à Harvard et cofondateur du psychédélisme avec le « pape du LSD » Timothy Leary avant de vendre cette autobiographie spirituelle à plus d'un million d'exemplaires, de fonder plusieurs centres consacrés à la spiritualité, d'écrire d'autres ouvrages, de faire des conférences à l'internationale... Il a rencontré Eckhart Tolle le 28 octobre 2011 à Hawaï pour dialoguer sur le réveil spirituel et la transformation de la conscience. Le public pouvait y assister en payant $\$ 100$ ou $\$ 125$ (pour les meilleures 
« nouveau syncrétisme », appartenant à la « tradition originale et autonome » occultiste (Stoczkowski, 1999, p. 293), dans le sens où le New Age tente de synthétiser modernité et tradition, conscient et inconscient, culture et nature, psychologie et physiologie, athéisme et théosophie, science et religion...

Si on fait l'hypothèse de considérer que la religion (les religions) a d'abord imposé une thèse sur le sens de l'existence et du monde qui a été remise en question par une antithèse scientifique, le New Age tente, quant à lui, de faire une synthèse des deux visions par un travail de double « désaliénation culturelle » (Rivière, 2008, p. 191), une " libération globale face à une aliénation globale » (Desroches, 1973, p. 137), vis-à-vis de la culture scientifique et de la culture monothéiste. Alors que la culture scientifique est une vaste entreprise analytique, le New Age tente de synthétiser, tout en les modifiant, les idées, philosophies, spiritualités, ésotérismes, croyances. Et plutôt que de rejeter les religions dans leur totalité, le New Age préfère les unifier, ou du moins sélectionner au sein d'elles (les croyances et pratiques orthodoxes) ou autour d'elles (les croyances et pratiques hétérodoxes) ce qui peut être utile pour construire ce mouvement en l'adaptant à la modernité. Certains dogmes, rites, symboles... chrétiens sont donc conservés.

Ce besoin d'enchantement des new agers va jusqu'au fait d'enchanter les religions elles-mêmes. A la manière de Dan Brown dans le fameux roman ésotérique $\mathrm{Da}$ Vinci Code où les enquêteurs parviennent à découvrir une histoire alternative au christianisme officiel, le New Age revisite l'histoire des religions et contribue ainsi à les resacraliser et réenchanter en réécrivant la Bible à travers ce qu'on appelle les Actes, Apocalypses, Évangiles apocryphes dont la non-authentification et la non-reconnaissance par l'Église catholique donne un aspect mystérieux, hétérodoxe et sacré.

De même, les six apparitions mariales « observées » par trois enfants de Fatima au Portugal en 1917 seraient en fait des OVNI extraterrestres. Ces mêmes extraterrestres qui seraient à l'origine d'une grande partie des complots politiques, militaires, scientifiques et économiques qui ont irrigué l'histoire humaine (Poulain, 2010). Mais insérés dans le prisme idéologique de la " pensée positives » New Age, ces extraterrestres seraient aussi possiblement les sauveurs de l'humanité. En effet, les humains ne parvenant qu'à s'entretuer (crises miliaires, nucléaires,

places) ou simplement regarder Eckhart Tolle TV (\$14,95/mois pour du streaming). 
écologiques...) et la minorité d'entre eux qui revendique des pouvoirs supranormaux - médiums, astrologues, enfants indigos, sages spirituels, voyants, télépathes, géomanciens, magnétiseurs, radiesthésistes, chamans... - n'étant pas assez nombreux et puissants pour inverser cette tendance, les messies extraterrestres - ces êtres forcément meilleurs et supérieurs aux humains intellectuellement, technologiquement, moralement, spirituellement puisqu'ils sont capables de venir sur terre sans y annihiler ou asservir sa population - peuvent être considérés comme l'ultime chance de survie terrienne.

Le New Age reconfigure et réinvente donc le religieux en puisant sélectivement dans un stock de croyances et pratiques religieuses, philosophiques, spirituelles, ésotériques. Il constitue ou renforce une mythologie moderne alternative aux mythologies judéo-chrétiennes et scientifico-rationnelles, et faite de calendriers mayas, d'enlèvements extraterrestres, de confréries secrètes, de dossiers gouvernementaux classés, de cités souterraines, de visiteurs cosmiques, d'êtres de lumière, de dimensions multiples, d'architecture cyclopéenne préhistorique...

Le récit New Age va aussi à l'encontre du récit moderne rationaliste et matérialiste des scientifiques, bien qu'il en soit en partie issu, qu'il l'ait intériorisé et le reproduise sans en avoir forcément conscience. Comme dans le récit moderne, le récit New Age tente de rationnaliser la société, c'est-à-dire d'expliquer ses conditions de possibilité. Mais dans le récit New Age, la science doit servir avant tout à expliquer le religieux de la même façon que le religieux doit expliquer la science (Champion, 1993). En effet, ce récit spiritualise le matériel (néopaganisme) et matérialise le spirituel (mediumnisme). Le rationnel doit servir l'irrationnel sans « tomber » dans le « scientisme matérialiste » «borné » et « sectaire » (termes utilisés par les new agers pour déplorer le scepticisme systématique des scientifiques).

Ainsi, beaucoup d'animateurs, invités et auditeurs se passionnent pour l'extraordinaire, l'invisible, le mystère, l'étrange, l'inconnu, le « merveilleux » (Renard, 2011) - c'est-à-dire des formes de sacralités alternatives aux religions monothéistes -, tout en se vantant souvent d'avoir une approche cartésienne et rationnelle. Il s'agit de réenchanter le monde en faisant parler les choses, la faune, la flore, la politique, le religieux, les morts ${ }^{1}$ grâce à la science car celle-ci doit avoir pour but de découvrir « l'énergie non matérielle et non physique ou uniquement

1 Les morts ne sont plus vraiment morts puisqu'ils continuent de communiquer avec nous grâce au spiritisme ou se réincarnent grâce au bouddhisme. 
physique » qui gouverne les choses, selon un auditeur. En effet, le New Age part du postulat que tout est vivant, tout est animé, tout a une âme (du latin anima), tout parle, tout fait sens, tout communique, tout est lié si on change de manière de penser le monde. Le New Age se présente comme une science spirituelle ou une spiritualité scientifique. Contrairement à l'idéologie scientifique qui niait tout fondement aux croyances religieuses, le New Age cherche un fondement scientifique à la spiritualité (grâce à des preuves empiriques archéologiques, textuelles, matérielles et des théories de grands scientifiques comme la relativité générale et la mécanique quantique d'Einstein, le paradoxe de non-séparabilité du théorème de Bell, le principe d'incertitude d'Heisenberg) et un fondement spirituel à la science (grâce à l'élaboration de constructions métaphysiques, anthropologies, théologies, théogonies, cosmogonies complexes). C'est pour cela que la spiritualité et l'occultisme se présentent souvent sous la forme de « para-science», voire de science.

\section{Conclusion}

La fameuse «mort de Dieu » nietzschéenne n'est aucunement la mort du religieux : «Pas plus qu'il n'y a de religion sans société, il n'y a de société sans religion : une société d'athées serait sans doute une société sans dieu(x), mais il ne s'ensuit pas qu'elle serait sans religion ni croyance. » (Perrot et al., 1992, p. 31). Le religieux, comme le politique, se redéploie, se redistribue, se réagence et s'actualise en permanence en fonction de nos récits qui sont aussi nombreux que nos manières de raconter et donner sens à la réalité, du fait des évolutions collectives (environnementales, sociales, politiques et économiques) et individuelles (les reconfigurations amicales, familiales, professionnelles en fonction de l'âge, des expériences et des capitaux socio-culturels et politico-économiques). Les hommes réinventent constamment d'autres formes d'action et de narration en relation avec d'autres formes de sacralité qui sont autant de formes de réenchantement. Lorsqu'ils sont confrontés à des formes de récit désenchantantes ou lorsque des formes de récit s'étiolent parce qu'elles perdent en légitimité, en intensité et en attractivité, donc en prégnance sur le social, les hommes ne tardent pas à « bricoler » aussitôt de nouvelles formes de récits réenchantantes, concurrentes et innovantes.

La question, selon Claude Rivière, est donc de savoir « comment s'entrelacent magie, religion et science, et pourquoi les recours à la magie s'amplifient dans un monde trop désenchanté pour n'être pas 
avide d'ésotérisme et de religion. » (2008, p. 133). Danièle HervieuLéger ne dit pas autre chose lorsqu'elle conseille la « sociologie de la modernité religieuse » dans son travail de recherche sur la croyance, et qui pourrait être appliqué à la sociologie appliquant le concept de réenchantement politique et religieux dans la mesure où la croyance joue un rôle fondamental dans ce concept : il s'agit « d'essayer de comprendre ensemble le mouvement par lequel la modernité continue de saper la crédibilité de tous les systèmes religieux, et celui par lequel elle fait en même temps surgir de nouvelles formes de croyance » car la sécularisation, loin d'être processus linéaire d' « athéisation », consiste en « l'ensemble des processus de réaménagements des croyances qui se produisent dans une société dont le moteur est l'inassouvissement des attentes qu'elle suscite, et dont la condition quotidienne est l'incertitude liée à la recherche interminable des moyens de les satisfaire. » (Hervieu-Léger, 1999, p. 42).

Loin d'une "fin de l'histoire " sans cesse annoncée et repoussée (Hegel, Kojève, Abellio, Fukuyama), les hommes aiment imaginer des récits réactionnaires ou révolutionnaires, ascétiques ou hédonistes, orthodoxes ou libéraux pour construire des réalités alternatives réenchantées.

Ainsi, le New Age est une tentative de réenchantement du récit à travers une nouvelle forme de récit utopique visant à changer la direction prise par l'humanité. Il vise à révolutionner la société, du moins à la modifier radicalement car la révolution est essentiellement intellectuelle, conceptuelle, spirituelle et individuelle. Il s'agit d' « éveiller les consciences » à la manière des « révolutions minuscules » (Chancel \& Tixier, 1981).

RIM est 1'un des nombreux supports de ce changement social et symbolique. Elle propose un modèle de radio alternative et militante qui promeut les nouveaux mouvements sociaux, artistiques, informatiques, contreculturels aussi bien que des nouvelles formes de croyances religieuses de type New Age. Tel « un système à produire du sens » alternatif (Laplantine, 1985, p. 169), cette communauté radiophonique nourrit d'autres formes de narrations utopiques donc enchantantes, comme l'explique un auditeur :

Quand on écoute Ici et Maintenant, assez vite, on entend des discours qui vont à l'encontre, qui vont gratter nos convictions nos représentations du monde et qui nous obligent à avoir un regard différent sur ce qui nous entoure. 


\section{Références}

Bourdieu, P. (1971). Genèse et structure du champ religieux. Revue française de sociologie, 12(3).

Champion, F. (1993). La Croyance en l'Alliance de la science et de la religion dans les nouveaux courants mystiques et ésotériques. ASSR, 82 .

Chancel, J., \& Tixier, P.-E. (Eds.). (1981). Les révolutions minuscules. Actions et causes en miettes. Qui s'engage aujourd'hui et pour quoi faire?, Paris : Autrement, 29.

Deleu, C. (2006). Les anonymes à la radio. Usages, fonctions et portée de la parole. Paris : De Boeck.

Derville, G. (1995). Les différents rôles du Bébête show auprès de ses téléspectateurs. Réseaux, 74.

Desroches, H. (1973). Sociologie de l'espérance et espérances des sociologies. Paris : Calmann-Lévy.

Dozon, J.-P. (2008). L'Afrique à Dieu et à Diable. États, ethnies et religions. Paris : Éditions Ellipses.

Ferreux, M.-J. (2000). Le New-Age, Ritualités et mythologies contemporaines. Paris : L'Harmattan.

Gauthier, A. (1996). Du visible au visuel. Paris : PUF.

Hervieu-Léger, D. (1999). Le pèlerin et le converti. La religion en mouvement. Paris : Flammarion.

Laplantine, F. (1985). La voyance comme mode de communication. In F. Laplantine (Ed.), Un voyant dans la ville : étude anthropologique d'un cabinet de consultation d'un voyant contemporain. Paris : Payot.

Perrot, M.-D., Rist, G. \& Sabelli, F. (1992). La mythologie programmée. L'économie des croyances dans la société moderne. Paris: PUF.

Poulain, S. (en cours de publication (a)). Sens dessus dessous : « démocratisation » du religieux à Radio Ici et Maintenant. In H. Abdelkefi (Ed.), La crise du sens, Sfax : Publications de l'ERCILIS.

Poulain, S. (en cours de publication (b)). Postradio et postmodernité : contexte, enjeux et limites. In J.-J. Cheval (Ed.), Vers la Post Radio - Enjeux des mutations des objets et formes radiophoniques. Paris.

Poulain, S. (en cours de publication (c)). Les radios libres ou la diabolisation de la FM : qu'est-ce que libérer la parole veut dire ?. In T. Lefebvre \& S. Poulain (Éd.), Radios libres, 30 ans de FM : la parole libérée ?, Paris : INA/L'Harmattan.

Poulain, S. (2008). Guérir de la société grâce à la radio : usages des libres antennes de Radio Ici et Maintenant. In Actes des travaux du groupe de travail « Sociologie de la communication 》, Congrès AISLF. Disponible à : http://w3.aislf.univ-tlse2.fr/gtsc/ DOCS_SOCIO/istambul/Actes_AISLF_GT13_Istambul_2008.pdf

Poulain, S. (2010). La fabrique des extraterrestres, Mots, 92, Disponible à : http://mots. revues.org/index19401.html

Renard. J.-B. (2011). Le Merveilleux. Sociologie de l'extraordinaire. Paris : CNRS Éditions.

Rivière, C. (2008). Socio-anthropologie des religions. Armand Colin : Paris.

Sorbets, C. (2003). Écoute la radio la nuit : écoute en veilleuse ou écoute éveilleuse. In J.-J. Cheval (Ed.), Audiences, publics et pratiques radiophoniques, Pessac : MSHA.

Stoczkowski, W. (1999). Des Hommes, des dieux et des extraterrestres. Ethnologie d'une croyance moderne. Paris : Flammarion. 